\title{
Multiple positive almost periodic solutions for some nonlin- ear integral equations
}

\author{
Hui-Sheng Ding ${ }^{\mathrm{a}, *}$, Juan J. Nieto ${ }^{\mathrm{b}}$, Qiu-Feng Zou \\ ${ }^{a}$ College of Mathematics and Information Science, Jiangxi Normal University, Nanchang, Jiangxi 330022, People's Republic of China. \\ ${ }^{b}$ Departamento de Estadística, Análisis Matemático y Optimización, Facultad de Matemáticas, Universidad de Santiago de Compostela, \\ 15782, Santiago de Compostela, Spain.
}

Communicated by R. Saadati

\begin{abstract}
This paper is concerned with the existence of multiple positive almost periodic solutions for a nonlinear integral equation. By using Avery-Henderson and Leggett-Williams multiple fixed point theorems on cones, the existence theorems of multiple positive almost periodic solutions for the addressed integral equation are established under some sufficient assumptions. An example is given to illustrate our results.
\end{abstract}

Keywords: Almost periodic, multiple solutions, integral equation.

2010 MSC: 45G10, 34K14.

(C)2018 All rights reserved.

\section{Introduction and preliminaries}

Recently, the existence of almost periodic type solutions for various kinds of integral equations has been of great interest for many authors (see, e.g., [1-6, 8, 10-13, 15, 17, 18] and references therein). Especially, in [1, 17], Agarwal, $\mathrm{O}^{\prime}$ Regan, and Meehan studied the existence of single or multiple almost periodic solutions for the following Fredholm integral equation:

$$
y(t)=h(t)+\int_{\mathbb{R}} k(t, s) f(s, y(s)) d s, \quad t \in \mathbb{R} .
$$

Stimulated by [1, 17], in this paper, we aim to investigate the existence of multiple positive almost periodic solutions for the following more general integral equation:

$$
y(t)=e(t, y(t))\left[h(t)+\int_{\mathbb{R}} k(t, s) f(s, y(s)) d s\right], \quad t \in \mathbb{R} .
$$

\footnotetext{
${ }^{*}$ Corresponding author

Email addresses: dinghs@mail .ustc.edu.cn (Hui-Sheng Ding), juanjose.nieto.roig@usc.es (Juan J. Nieto), 642074260@qq. com (Qiu-Feng Zou)
}

doi: $10.22436 /$ jnsa.011.05.11

Received: 2016-11-23 Revised: 2018-02-12 Accepted: 2018-02-15 
As for the existence of single periodic and almost periodic solutions, the authors in $[10,11,15,16]$ have made extensive studies on equation (1.2) and its variants. However, it seems that there is few result concerning multiple periodic and almost periodic solutions for equation (1.2). In fact, to the best of our knowledge, even for equation (1.1), the work of Agarwal and O'Regan [1] is the only known result about multiple periodic and almost periodic solutions. That is another motivation of this work. We will utilize Avery-Henderson and Leggett-Williams multiple fixed point theorems on cones to obtain our main results. This is different from [1], where "single fixed point" theorem was used twice to obtain the existence of two periodic and almost periodic solutions.

Throughout the rest of this paper, if there is no special statement, we denote by $\mathbb{R}$ the set of real numbers, by $X$ a Banach space, by $C(\mathbb{R}, X)$ the set of all continuous functions from $\mathbb{R}$ to $X$, and denote by $\mathfrak{L i p}\left(\mathbb{R} \times \mathbb{R}, \mathbb{R}^{+}\right)$the set of all functions $f: \mathbb{R} \times \mathbb{R} \rightarrow \mathbb{R}^{+}$satisfying that there exists a constant $\mathrm{L}>0$ such that

$$
|f(t, x)-f(t, y)| \leqslant L|x-y|, \quad t \in \mathbb{R}, x, y \in \mathbb{R} .
$$

Moreover, for every $f \in \mathfrak{L i p}\left(\mathbb{R} \times \mathbb{R}, \mathbb{R}^{+}\right)$,

$$
L_{f}:=\sup _{t \in \mathbb{R}, x \neq y} \frac{|f(t, x)-f(t, y)|}{|x-y|} .
$$

Next, let us recall some notations about cones and two fixed point theorems. For more details, we refer the reader to $[7,14]$.

Let $X$ be a real Banach space. A closed convex set $\mathrm{K}$ in $\mathrm{X}$ is called a cone if the following conditions are satisfied:

(i) if $x \in K$, then $\lambda x \in K$ for any $\lambda \geqslant 0$;

(ii) if $x \in K$ and $-x \in K$, then $x=0$.

A non-negative continuous functional $\psi$ is said to be a concave on $K$ if $\psi$ is continuous and

$$
\psi(\mu x+(1-\mu) y) \geqslant \mu \psi(x)+(1-\mu) \psi(y), \quad x, y \in K, \mu \in[0,1] .
$$

Letting $c_{1}, c_{2}, c_{3}$ be three positive constants, and $\phi$ be a nonnegative continuous functional on $K$, we denote

$$
\begin{array}{rlrl}
\mathrm{K}_{\mathrm{c}_{1}} & =\left\{y \in \mathrm{K}:\|y\|<\mathrm{c}_{1}\right\}, & \overline{\mathrm{K}_{\mathrm{c}_{1}}} & =\left\{\mathrm{y} \in \mathrm{K}:\|y\| \leqslant \mathrm{c}_{1}\right\}, \\
\mathrm{K}\left(\phi, \mathrm{c}_{1}\right) & :=\left\{x \in \mathrm{K}: \phi(x)<\mathrm{c}_{1}\right\}, & \overline{\mathrm{K}\left(\phi, \mathrm{c}_{1}\right)}:=\left\{x \in \mathrm{K}: \phi(x) \leqslant \mathrm{c}_{1}\right\}, \\
\partial \mathrm{K}\left(\phi, \mathrm{c}_{1}\right) & :=\left\{x \in \mathrm{K}: \phi(x)=\mathrm{c}_{1}\right\}, & \mathrm{K}\left(\phi, \mathrm{c}_{2}, \mathrm{c}_{3}\right) & =\left\{\mathrm{y} \in \mathrm{K}: \mathrm{c}_{2} \leqslant \phi(y),\|\mathrm{y}\| \leqslant \mathrm{c}_{3}\right\} .
\end{array}
$$

In addition, we call that $\phi$ is increasing on $K$ if $\phi(x) \geqslant \phi(y)$ for all $x, y \in K$ with $x-y \in K$.

Lemma 1.1 ([7]). Let $\mathrm{K}$ be a cone in a real Banach space $\mathrm{X}, \alpha$ and $\varphi$ be two increasing, nonnegative, and continuous functionals on $\mathrm{K}$, and $\rho$ be a nonnegative continuous functional on $\mathrm{K}$ with $\rho(0)=0$ such that for some $\mathrm{c}>0$ and $M>0$,

$$
\varphi(x) \leqslant \rho(x) \leqslant \alpha(x), \quad\|x\| \leqslant M \varphi(x), \quad x \in \overline{\mathrm{K}(\varphi, c)} .
$$

Moreover, suppose that there exists a completely continuous operator $\Phi: \overline{\mathrm{K}(\varphi, \mathrm{c})} \rightarrow \mathrm{K}$ and $0<\mathrm{a}<\mathrm{b}<\mathrm{c}$ such that

$$
\rho(\lambda x) \leqslant \lambda \rho(x), \quad 0 \leqslant \lambda \leqslant 1, x \in \partial K(\rho, b),
$$

and

(i) $\varphi(\Phi x)>c$ for all $x \in \partial \mathrm{K}(\varphi, \mathrm{c})$;

(ii) $\rho(\Phi x)<b$ for all $x \in \partial K(\rho, b)$;

(iii) $\mathrm{K}(\alpha, \mathrm{a}) \neq \emptyset$ and $\alpha(\Phi x)>$ a for all $x \in \partial \mathrm{K}(\alpha, \mathrm{a})$. 
Then $\Phi$ has at least two fixed points $\mathrm{x}_{1}, \mathrm{x}_{2}$ belonging to $\overline{\mathrm{K}(\varphi, \mathrm{c})}$ such that

$$
a<\alpha\left(x_{1}\right), \rho\left(x_{1}\right)<b, \text { and } b<\rho\left(x_{2}\right), \varphi\left(x_{2}\right)<c .
$$

Lemma 1.2 ([14]). Let $\mathrm{K}$ be a cone in a real Banach space $\mathrm{X}, \mathrm{c}_{4}$ be a positive constant, $\Phi: \overline{\mathrm{K}_{\mathrm{c}_{4}}} \rightarrow \overline{\mathrm{K}_{\mathrm{c}_{4}}}$ be a completely continuous mapping, and $\psi$ be a concave nonnegative continuous functional on $\mathrm{K}$ with $\psi(\mathrm{u}) \leqslant\|\mathrm{u}\|$ for all $\mathrm{u} \in \overline{\mathrm{K}_{\mathrm{c}_{4}}}$. Suppose that there exist three constants $\mathrm{c}_{1}, \mathrm{c}_{2}, \mathrm{c}_{3}$ with $0<\mathrm{c}_{1}<\mathrm{c}_{2}<\mathrm{c}_{3} \leqslant \mathrm{c}_{4}$ such that

(i) $\left\{\mathrm{u} \in \mathrm{K}\left(\psi, \mathrm{c}_{2}, \mathrm{c}_{3}\right): \psi(\mathrm{u})>\mathrm{c}_{2}\right\} \neq \varnothing$, and $\psi(\Phi \mathrm{u})>\mathrm{c}_{2}$ for all $\mathrm{u} \in \mathrm{K}\left(\psi, \mathrm{c}_{2}, \mathrm{c}_{3}\right)$;

(ii) $\|\Phi u\|<c_{1}$ for all $\mathrm{u} \in \overline{\mathrm{K}_{\mathrm{c}_{1}}}$;

(iii) $\psi(\Phi u)>c_{2}$ for all $u \in K\left(\psi, c_{2}, c_{4}\right)$ with $\|\Phi u\|>c_{3}$.

Then $\Phi$ has at least three fixed points $\mathfrak{u}_{1}, \mathfrak{u}_{2}, \mathfrak{u}_{3}$ in $\overline{\mathrm{k}_{\mathbf{c}_{4}}}$. Furthermore, $\left\|\mathfrak{u}_{1}\right\|<\mathrm{c}_{1}<\left\|\mathfrak{u}_{2}\right\|$, and $\psi\left(\mathfrak{u}_{2}\right)<\mathrm{c}_{2}<\psi\left(\mathfrak{u}_{3}\right)$.

We also need to recall some notations and properties about almost periodic functions and equi-almost periodic functions. For more details, we refer the reader to [9].

Definition 1.3. A set $E \subset \mathbb{R}$ is called relatively dense if there exists a number $l>0$ such that

$$
(a, a+l) \cap E \neq \emptyset
$$

for every $a \in \mathbb{R}$.

Definition 1.4. A function $f \in C(\mathbb{R}, X)$ is called almost periodic if for every $\varepsilon>0$ there exists a relatively dense set $T(f, \varepsilon) \subset \mathbb{R}$ such that

$$
\|f(t+\tau)-f(t)\|<\varepsilon
$$

for all $t \in \mathbb{R}$ and $\tau \in T(f, \varepsilon)$. We denote the set of all such functions by $A P(\mathbb{R}, X)$. Also, we denote $\operatorname{AP}(\mathbb{R}, \mathbb{R})$ by $A P(\mathbb{R})$ for convenience.

Definition 1.5. A set $F \subset C(\mathbb{R}, X)$ is called equi-almost periodic if for every $\varepsilon>0$, there exists a relatively dense set $T(F, \varepsilon) \subset \mathbb{R}$ such that

$$
\|f(t+\tau)-f(t)\|<\varepsilon
$$

for all $f \in F, t \in \mathbb{R}$, and $\tau \in T(F, \varepsilon)$.

Definition 1.6. Let $\Omega \subset X$. A continuous function $f: \mathbb{R} \times \Omega \rightarrow X$ is called almost periodic in $t$ uniformly for $x \in \Omega$ if for every $\varepsilon>0$ and for every compact subset $K \subset \Omega$, there exists a relatively dense set $T(f, K, \varepsilon)$ such that

$$
\|f(t+\tau, x)-f(t, x)\|<\varepsilon \quad \text { for all } t \in \mathbb{R}, x \in K, \tau \in T(f, K, \varepsilon) .
$$

We denote by $\operatorname{AP}(\mathbb{R} \times \Omega, X)$ the set of all such functions.

Remark 1.7. It is easy to see that $f \in \operatorname{AP}(\mathbb{R} \times \Omega, X)$ is equivalent to the fact that for every compact subset $\mathrm{K} \subset \Omega,\{f(\cdot, x)\}_{x \in \mathrm{K}}$ is equi-almost periodic.

Lemma 1.8 ([9]). The following assertions hold true:

(a) $\operatorname{AP}(\mathbb{R}, X)$ is Banach space under the supremum norm;

(b) $f, g \in A P(\mathbb{R}, \mathbb{R})$ implies that $f \cdot g \in A P(\mathbb{R}, \mathbb{R})$;

(c) $\mathrm{f} \in \mathrm{AP}(\mathbb{R} \times \Omega, X)$ and $\mathrm{g} \in \mathrm{AP}(\mathbb{R}, \mathrm{X})$ imply that $\mathrm{f}(\cdot, \mathrm{g}(\cdot)) \in \mathrm{AP}(\mathbb{R}, \mathrm{X})$ provided that $\overline{\{\mathrm{g}(\mathrm{t}): \mathrm{t} \in \mathbb{R}\}} \subset \Omega$;

(d) every finite set $\mathrm{F} \subset \mathrm{AP}(\mathbb{R}, \mathrm{X})$ is equi-almost periodic.

Lemma 1.9 ([9]). The necessary and sufficient condition that $\mathcal{F} \subset A \mathrm{P}(\mathbb{R}, \mathrm{X})$ be precompact is that the following properties hold true:

(i) for every $t \in \mathbb{R},\{f(t): f \in \mathcal{F}\}$ is precompact in $X$;

(ii) $\mathcal{F}$ is equi-continuous and equi-almost periodic.

Moreover, we clarify what is called Carathéodory function.

Definition 1.10. A function $f: \mathbb{R} \times \mathbb{R} \rightarrow \mathbb{R}$ is called a Carathéodory function if the map $t \rightarrow f(t, u)$ is measurable for all $u \in \mathbb{R}$, and the map $u \rightarrow f(t, u)$ is continuous for almost all $t \in \mathbb{R}$. 


\section{Multiple almost periodic solutions}

Throughout the rest of this paper, let $1 \leqslant p \leqslant \infty$, q be such that $\frac{1}{p}+\frac{1}{q}=1$, and $\xi, \eta, c, M$ be fixed positive constants. Moreover, assume that

$$
f(t, x)=\xi \widetilde{f}(t, x)+g(t, x), \quad t, x \in \mathbb{R},
$$

where $\tilde{f}, g: \mathbb{R} \times \mathbb{R} \rightarrow \mathbb{R}^{+}$are two Carathéodory functions satisfying the following assumptions:

(H0) $e \in A P(\mathbb{R} \times \mathbb{R}, \mathbb{R}) \cap \mathfrak{L i p}\left(\mathbb{R} \times \mathbb{R}, \mathbb{R}^{+}\right)$and $0<\inf _{\mathrm{t} \in \mathbb{R}, x \in \mathbb{R}^{+}} e(t, x) \leqslant \sup _{\mathrm{t} \in \mathbb{R}, x \in \mathbb{R}^{+}} e(t, x)<+\infty$.

(H1) For every $r>0$, there exists a function $\mu_{r} \in L^{p}(\mathbb{R})$ such that $|x| \leqslant r$ implies that $\tilde{f}(t, x) \leqslant \mu_{r}(t)$ for almost all $t \in \mathbb{R}$. In addition, there exists $g \in L^{p}(\mathbb{R})$ such that $g(t, x) \geqslant \eta g(t)$ for all $x \geqslant c$ and almost all $t \in \mathbb{R}$, and $g(t, x) \leqslant M g(t)$ for all $x \in \mathbb{R}$ and almost all $t \in \mathbb{R}$.

(H2) Let $k: \mathbb{R} \times \mathbb{R} \rightarrow \mathbb{R}^{+}$be such that (i) there exists a function $m \in L^{\mathrm{q}}(\mathbb{R})$ such that $|k(t, s)| \leqslant m(s)$ for all $t \in \mathbb{R}$ and almost all $s \in \mathbb{R}$; (ii) the map $t \rightarrow k(t, \cdot)$ is an almost periodic function from $\mathbb{R}$ to $\mathrm{L}^{\mathrm{q}}(\mathbb{R})$.

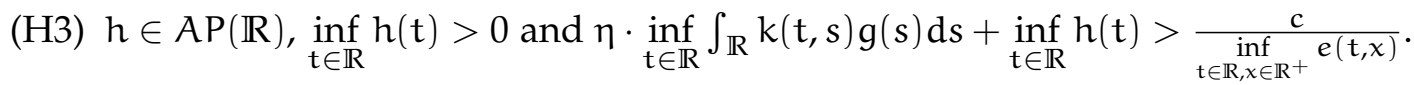

(H4) There exists $b \in(0, c)$ such that $g(t, x) \leqslant g(t)$ for all $x \leqslant b$ and almost all $t \in \mathbb{R}$, and

$$
\|h\|+\xi\|m\|_{q}\left\|\mu_{b}\right\|_{p}+\|m\|_{q}\|g\|_{p}<\frac{b}{\sup _{t \in \mathbb{R}, x \in \mathbb{R}^{+}} e(t, x)} .
$$

Theorem 2.1. Assume that (H0)-(H4) hold. Then, there exists $\mathrm{L}^{*}>0$ such that equation (1.2) has at least two positive almost periodic solutions provided that $\mathrm{L}_{e}<\mathrm{L}^{*}$ and

$$
\lim _{r \rightarrow+\infty} \frac{\left\|\mu_{r}\right\|_{p}}{r}=0
$$

Proof. Since

$$
\lim _{\sigma \rightarrow 0} \sigma\left\|\mu_{\frac{c}{\sigma}}\right\|_{p}=0, \quad \inf _{t \in \mathbb{R}} h(t)>0,
$$

and

$$
0<\inf _{t \in \mathbb{R}, x \in \mathbb{R}^{+}} e(t, x) \leqslant \sup _{t \in \mathbb{R}, x \in \mathbb{R}^{+}} e(t, x)<+\infty,
$$

one can choose $\sigma \in(0,1)$ such that

$$
\sigma\|h\|+\sigma \cdot M\|m\|_{q}\|g\|_{p}+\sigma \cdot \xi\|m\|_{q}\left\|\mu_{\frac{c}{\sigma}}\right\|_{p} \leqslant \inf _{t \in \mathbb{R}} h(t) \cdot \frac{\inf _{t \in \mathbb{R}, x \in \mathbb{R}^{+}} e(t, x)}{\sup _{t \in \mathbb{R}, x \in \mathbb{R}^{+}} e(t, x)} .
$$

Now, we introduce a set

$$
K=\left\{u \in A P(\mathbb{R}): \inf _{t \in \mathbb{R}} u(t) \geqslant \sigma\|u\|\right\} .
$$

It is not difficult to verify that $K$ is a cone in $A P(\mathbb{R})$. Denote

$$
\varphi(\mathfrak{u})=\inf _{\mathfrak{t} \in \mathbb{R}} u(t), \quad \rho(u)=\alpha(\mathfrak{u})=\|u\|, \quad u \in K .
$$

It is clear that $\varphi, \rho$ and $\alpha$ are increasing, nonnegative, and continuous functionals on $\mathrm{K}$ with $\rho(0)=0$. Moreover, we have

$$
\|u\| \leqslant \frac{1}{\sigma} \inf _{\mathfrak{t} \in \mathbb{R}} u(t)=\sigma^{-1} \varphi(u), \quad \rho(\lambda u)=\lambda \rho(u)
$$

for all $u \in K$ and $0 \leqslant \lambda \leqslant 1$. 
Fix $y \in \overline{\mathrm{K}(\varphi, c)}$. Define an operator $\Phi_{y}$ on $\operatorname{AP}(\mathbb{R})$ by

$$
\left(\Phi_{y} u\right)(t)=e(t, u(t))\left[h(t)+\int_{\mathbb{R}} k(t, s) f(s, y(s)) d s\right], \quad t \in \mathbb{R}, u \in A P(\mathbb{R}) .
$$

By (H1) and (H2), it is not difficult to see that $t \rightarrow \int_{\mathbb{R}} k(t, s) f(s, y(s))$ ds belongs to $A P(\mathbb{R})$. Then, noting that $h \in A P(\mathbb{R})$ and $e(\cdot, u(\cdot)) \in A P(\mathbb{R})$ for every $u \in A P(\mathbb{R})$, by Lemma 1.8 , we conclude that $\Phi_{y}$ maps $\operatorname{AP}(\mathbb{R})$ into $A P(\mathbb{R})$. Moreover, for every $u, v \in A P(\mathbb{R})$, by using $(\mathrm{H} 1)$ and the fact that $\|y\| \leqslant \frac{c}{\sigma}$ since $\mathrm{y} \in \overline{\mathrm{K}(\varphi, \mathrm{c})}$, we have

$$
\begin{aligned}
\left|\left(\Phi_{y} u\right)(t)-\left(\Phi_{y} v\right)(t)\right| & \leqslant L_{e}|u(t)-v(t)| \cdot\left|h(t)+\int_{\mathbb{R}} k(t, s) f(s, y(s)) d s\right| \\
& \leqslant L_{e}\|u-v\| \cdot\left[\|h\|+\int_{\mathbb{R}} m(s)\left(\xi \mu_{\frac{c}{\sigma}}(s)+M g(s)\right) d s\right] \\
& \leqslant L_{e}\|u-v\| \cdot\left[\|h\|+\xi\left\|\mu_{\frac{c}{\sigma}}\right\|_{p}\|m\|_{q}+M\|g\|_{p}\|m\|_{q}\right] .
\end{aligned}
$$

Letting $\mathrm{L}^{*}=\frac{1}{\|\mathrm{~h}\|+\xi\left\|\mu_{\frac{c}{\sigma}}\right\|_{p}\|m\|_{q}+M\|g\|_{p}\|m\|_{q}}$, we get

$$
\left\|\Phi_{y} u-\Phi_{y} v\right\| \leqslant \frac{\mathrm{L}_{e}}{\mathrm{~L}^{*}}\|\mathrm{u}-v\|
$$

which means that $\Phi_{y}$ has a unique fixed point $u_{y} \in A P(\mathbb{R})$ provided that $L_{e}<L^{*}$.

Now, define an operator $\Phi$ on $\overline{\mathrm{K}(\varphi, \mathrm{c})}$ by $\Phi y=u_{y}$, i.e.,

$$
(\Phi y)(t)=u_{y}(t)=e\left(t, u_{y}(t)\right)\left[h(t)+\int_{\mathbb{R}} k(t, s) f(s, y(s)) d s\right], \quad t \in \mathbb{R}, y \in \overline{K(\varphi, c)} .
$$

Next, let us verify all the assumptions of Lemma 1.1. First, let us prove that $\Phi$ maps $\overline{K(\varphi, c)}$ into K. From the above proof, we know that $\Phi y=u_{y} \in A P(\mathbb{R})$ for all $y \in \overline{\mathrm{K}(\varphi, c)}$. For every $y \in \overline{\mathrm{K}(\varphi, \mathrm{c})}$, since $\|y\| \leqslant \frac{c}{\sigma}$, by (H1) and (2.1), we get

$$
(\Phi u)(t) \geqslant \inf _{t \in \mathbb{R}, x \in \mathbb{R}^{+}} e(t, x) \cdot \inf _{t \in \mathbb{R}} h(t) \geqslant \sigma \cdot \sup _{t \in \mathbb{R}, x \in \mathbb{R}^{+}} e(t, x)\left[\|h\|+\|m\|_{q}\left(\xi\left\|\mu_{\frac{c}{\sigma}}\right\|_{p}+M\|g\|_{p}\right)\right] \geqslant \sigma\|\Phi u\|
$$

for all $t \in \mathbb{R}$. Thus, $\Phi u \in K$. This means that $\Phi$ is a mapping from $\overline{K(\varphi, c)}$ to $K$.

Next, let us show that $\Phi: \overline{\mathrm{K}(\varphi, \mathrm{c})} \rightarrow \mathrm{K}$ is completely continuous. Let $\mathrm{y}_{\mathrm{n}} \rightarrow \mathrm{y}$ in $\overline{\mathrm{K}(\varphi, \mathrm{c})}$. Noting that

$$
\begin{aligned}
& \left|\left(\Phi y_{n}\right)(t)-(\Phi y)(t)\right| \\
& =\left|e\left(t,\left(\Phi y_{n}\right)(t)\right)\left[h(t)+\int_{\mathbb{R}} k(t, s) f\left(s, y_{n}(s)\right) d s\right]-e(t,(\Phi y)(t))\left[h(t)+\int_{\mathbb{R}} k(t, s) f(s, y(s)) d s\right]\right| \\
& \leqslant\left|e\left(t,\left(\Phi y_{n}\right)(t)\right)\left[h(t)+\int_{\mathbb{R}} k(t, s) f\left(s, y_{n}(s)\right) d s\right]-e\left(t,\left(\Phi y_{n}\right)(t)\right)\left[h(t)+\int_{\mathbb{R}} k(t, s) f(s, y(s)) d s\right]\right| \\
& \quad+\left|e\left(t,\left(\Phi y_{n}\right)(t)\right)\left[h(t)+\int_{\mathbb{R}} k(t, s) f(s, y(s)) d s\right]-e(t,(\Phi y)(t))\left[h(t)+\int_{\mathbb{R}} k(t, s) f(s, y(s)) d s\right]\right| \\
& \leqslant\left|e\left(t,\left(\Phi y_{n}\right)(t)\right)\right| \int_{\mathbb{R}} k(t, s)\left|f\left(s, y_{n}(s)\right)-f(s, y(s))\right| d s+\frac{L_{e}}{L^{*}}\left|\left(\Phi y_{n}\right)(t)-(\Phi y)(t)\right| \\
& \leqslant \sup _{t \in \mathbb{R}, x \in \mathbb{R}^{+}} e(t, x) \int_{\mathbb{R}} m(s)\left|f\left(s, y_{n}(s)\right)-f(s, y(s))\right| d s+\frac{L_{e}}{L^{*}}\left\|\Phi y_{n}-\Phi y\right\|,
\end{aligned}
$$

which yields that

$$
\left\|\Phi y_{n}-\Phi y\right\| \leqslant \frac{L^{*}}{L^{*}-L_{e}} \cdot \sup _{t \in \mathbb{R}, x \in \mathbb{R}^{+}} e(t, x) \cdot \int_{\mathbb{R}} m(s)\left|f\left(s, y_{n}(s)\right)-f(s, y(s))\right| d s .
$$


Then, noting that $\left|f\left(s, y_{n}(s)\right)-f(s, y(s))\right| \leqslant 2 \mu_{\frac{c}{\sigma}}(s)$ for almost all $s \in \mathbb{R}$, by using the dominated convergence theorem, we conclude that $\Phi y_{n} \rightarrow \Phi y$. Thus, $\Phi$ is continuous. In order to show that $\Phi$ is compact, by Lemma 1.9, we only need to verify that $\{(\Phi y)(t): y \in \overline{\mathrm{K}(\varphi, c)}\}$ is uniformly bounded, equi-continuous, and equi-almost periodic. The uniform boundedness of $\{(\Phi y)(t): y \in \overline{K(\varphi, c)}\}$ obviously holds. For all $t_{1}, t_{2} \in \mathbb{R}$, and $y \in \bar{K}(\varphi, c)$, we have

$$
\begin{aligned}
\mid(\Phi y) & \left(t_{1}\right)-(\Phi y)\left(t_{2}\right) \mid \\
= & \left|e\left(t_{1},(\Phi y)\left(t_{1}\right)\right)\left[h\left(t_{1}\right)+\int_{\mathbb{R}} k\left(t_{1}, s\right) f(s, y(s)) d s\right]-e\left(t_{2},(\Phi y)\left(t_{2}\right)\right)\left[h\left(t_{2}\right)+\int_{\mathbb{R}} k\left(t_{2}, s\right) f(s, y(s)) d s\right]\right| \\
\leqslant & \left|e\left(t_{1},(\Phi y)\left(t_{1}\right)\right)\left[h\left(t_{1}\right)+\int_{\mathbb{R}} k\left(t_{1}, s\right) f(s, y(s)) d s\right]-e\left(t_{1},(\Phi y)\left(t_{1}\right)\right)\left[h\left(t_{2}\right)+\int_{\mathbb{R}} k\left(t_{2}, s\right) f(s, y(s)) d s\right]\right| \\
& +\left|e\left(t_{1},(\Phi y)\left(t_{1}\right)\right)\left[h\left(t_{2}\right)+\int_{\mathbb{R}} k\left(t_{2}, s\right) f(s, y(s)) d s\right]-e\left(t_{2},(\Phi y)\left(t_{2}\right)\right)\left[h\left(t_{2}\right)+\int_{\mathbb{R}} k\left(t_{2}, s\right) f(s, y(s)) d s\right]\right| \\
\leqslant & \sup _{t \in \mathbb{R}, x \in \mathbb{R}^{+}} e(t, x)\left[\left|h\left(t_{1}\right)-h\left(t_{2}\right)\right|+\int_{\mathbb{R}}\left|k\left(t_{1}, s\right)-k\left(t_{2}, s\right)\right| \mu_{\frac{c}{\sigma}}(s) d s\right] \\
& +\left|e\left(t_{1},(\Phi y)\left(t_{1}\right)\right)-e\left(t_{2},(\Phi y)\left(t_{2}\right)\right)\right| \cdot\left[\|h\|+\|m\|_{\mathfrak{q}}\left(\xi\left\|\mu_{\frac{c}{\sigma}}\right\|_{p}+M\|g\|_{p}\right)\right] \\
\leqslant & \sup _{t \in \mathbb{R}, x \in \mathbb{R}^{+}} e(t, x)\left[\left|h\left(t_{1}\right)-h\left(t_{2}\right)\right|+\left\|k\left(t_{1}, \cdot\right)-k\left(t_{2}, \cdot\right)\right\|_{\mathfrak{q}}\left\|\mu_{\frac{c}{\sigma}}\right\|_{p}\right] \\
& +\frac{L_{e}\left|(\Phi y)\left(t_{1}\right)-(\Phi y)\left(t_{2}\right)\right|+\left|e\left(t_{1},(\Phi y)\left(t_{1}\right)\right)-e\left(t_{2},(\Phi y)\left(t_{1}\right)\right)\right|}{L^{*}}
\end{aligned}
$$

which gives that

$$
\begin{aligned}
\left|(\Phi y)\left(t_{1}\right)-(\Phi y)\left(t_{2}\right)\right| \leqslant & \frac{\sup _{t \in \mathbb{R}, x \in \mathbb{R}^{+}} e(t, x) \cdot L^{*}}{L^{*}-L_{e}} \cdot\left[\left|h\left(t_{1}\right)-h\left(t_{2}\right)\right|+\left\|k\left(t_{1}, \cdot\right)-k\left(t_{2}, \cdot\right)\right\|_{q}\left\|\mu_{\frac{c}{\sigma}}\right\|_{p}\right] \\
& +\frac{\left|e\left(t_{1},(\Phi y)\left(t_{1}\right)\right)-e\left(t_{2},(\Phi y)\left(t_{1}\right)\right)\right|}{L^{*}-L_{e}} .
\end{aligned}
$$

Combining this with the fact that $h(t)$ is uniformly continuous on $\mathbb{R}$, the map $t \rightarrow k(t, \cdot)$ is uniformly continuous on $\mathbb{R}, e(\cdot, x)$ is uniformly continuous on $\mathbb{R}$ uniformly for $x$ belonging to bounded sets, we conclude that $\{(\Phi y)(t): y \in \bar{K}(\varphi, c)\}$ is equi-continuous. In addition, noting that $h \in A P(\mathbb{R})$, the map $t \rightarrow k(t, \cdot)$ is an almost periodic function from $\mathbb{R}$ to $\mathrm{L}^{\mathrm{q}}(\mathbb{R})$, and $e \in A \mathrm{P}(\mathbb{R} \times \mathbb{R}, \mathbb{R})$, it follows from (d) of Lemma 1.8 that for every $\varepsilon>0$,

$$
\mathrm{T}(\mathrm{h}, \varepsilon) \bigcap \mathrm{T}(\tilde{\mathrm{k}}, \varepsilon) \bigcap \mathrm{T}\left(e, \mathcal{R}_{\Phi}, \varepsilon\right)
$$

is relatively dense in $\mathbb{R}$, where

$$
\widetilde{k}(t)=k(t, \cdot), \quad \mathcal{R}_{\Phi}=\left[0, \sup _{t \in \mathbb{R}, y \in \bar{K}(\varphi, c)}(\Phi y)(t)\right] .
$$

Also, similar to the above proof of equi-continuity, one can obtain

$$
\begin{aligned}
& |(\Phi y)(t)-(\Phi y)(t+\tau)| \\
& \quad \leqslant \frac{\sup _{t \in \mathbb{R}, x \in \mathbb{R}^{+}} e(t, x) \cdot L^{*}}{L^{*}-L_{e}} \cdot\left[|h(t)-h(t+\tau)|+\|k(t, \cdot)-k(t+\tau, \cdot)\|_{q}\left\|\mu_{\frac{c}{\sigma}}\right\|_{p}\right] \\
& \quad+\frac{|e(t,(\Phi y)(t))-e(t+\tau,(\Phi y)(t))|}{L^{*}-L_{e}}, \quad t \in \mathbb{R}, \tau \in T(h, \varepsilon) \bigcap T(\widetilde{k}, \varepsilon) \bigcap T\left(e, \mathcal{R}_{\Phi}, \varepsilon\right),
\end{aligned}
$$


which means that $\{(\Phi y)(t): y \in \overline{\mathrm{K}(\varphi, \mathrm{c})}\}$ is equi-almost periodic. This completes the proof of $\Phi: \overline{\mathrm{K}(\varphi, \mathrm{c})} \rightarrow$ $\mathrm{K}$ being completely continuous.

It remains to show that the assumptions (i)-(iii) of Lemma 1.1 hold. Let $y \in \partial K(\varphi, c)$. Then, $y(t) \geqslant$ $\inf _{t \in \mathbb{R}} y(t)=\varphi(y)=c$ for all $t \in \mathbb{R}$, which means that $g(t, y(t)) \geqslant \eta g(t)$ for all $t \in \mathbb{R}$ by (H1). Combining this with (H3), we conclude that

$$
\begin{aligned}
\varphi(\Phi y) & =\inf _{t \in \mathbb{R}}\left(e\left(t, u_{y}(t)\right)\left[h(t)+\int_{\mathbb{R}} k(t, s) f(s, y(s)) d s\right]\right) \\
& \geqslant \inf _{t \in \mathbb{R}, x \in \mathbb{R}^{+}} e(t, x) \cdot\left[\inf _{t \in \mathbb{R}} \int_{\mathbb{R}} k(t, s) f(s, y(s)) d s+\inf _{t \in \mathbb{R}} h(t)\right] \\
& \geqslant \inf _{t \in \mathbb{R}, x \in \mathbb{R}^{+}} e(t, x) \cdot\left[\inf _{t \in \mathbb{R}} \int_{\mathbb{R}} k(t, s) g(s, y(s)) d s+\inf _{t \in \mathbb{R}} h(t)\right] \\
& \geqslant \inf _{t \in \mathbb{R}, x \in \mathbb{R}^{+}} e(t, x) \cdot\left[\eta \inf _{t \in \mathbb{R}} \int_{\mathbb{R}} k(t, s) g(s) d s+\inf _{t \in \mathbb{R}} h(t)\right]>c,
\end{aligned}
$$

which means that (i) of Lemma 1.1 holds. Let $y \in \partial K(\rho, b)$. Noting that $\|y\|=b$, by $(H 4), g(t, y(t)) \leqslant g(t)$ for all $t \in \mathbb{R}$. Then, again by (H4), we have

$$
\begin{aligned}
\rho(\Phi y) & =\sup _{t \in \mathbb{R}}\left|e\left(t, u_{y}(t)\right)\left[h(t)+\int_{\mathbb{R}} k(t, s) f(s, y(s)) d s\right]\right| \\
& \leqslant \sup _{t \in \mathbb{R}, x \in \mathbb{R}^{+}} e(t, x) \cdot\left[\|h\|+\int_{\mathbb{R}}|m(s)| \cdot\left|\xi \mu_{b}(s)+g(s)\right| d s\right] \\
& \leqslant \sup _{t \in \mathbb{R}, x \in \mathbb{R}^{+}} e(t, x) \cdot\left[\|h\|+\xi\|m\|_{q}\left\|\mu_{b}\right\|_{p}+\|m\|_{q}\|g\|_{p}\right]<b .
\end{aligned}
$$

This shows that (ii) of Lemma 1.1 holds. Finally, by choosing

$$
a=\frac{1}{2} \min \left\{\inf _{t \in \mathbb{R}, x \in \mathbb{R}^{+}} e(t, x) \cdot \inf _{t \in \mathbb{R}} h(t), b\right\}
$$

it is easy to verify (iii) of Lemma 1.1.

Now, by applying Lemma 1.1, there exist at least two fixed points $\mathfrak{u}_{1}, \mathfrak{u}_{2} \in \overline{\mathrm{K}(\varphi, c)}$, which satisfy

$$
u_{i}(t)=e\left(t, u_{i}(t)\right)\left[h(t)+\int_{\mathbb{R}} k(t, s) f\left(s, u_{i}(s)\right) d s\right], \quad t \in \mathbb{R}, i=1,2,
$$

i.e., $\mathfrak{u}_{1}, \mathfrak{u}_{2}$ are two almost periodic solutions to equation (1.2). Moreover, by Lemma 1.1, there holds

$$
\left\|u_{1}\right\|,\left\|u_{2}\right\| \geqslant a
$$

which yields that

$$
u_{1}(t), u_{2}(t) \geqslant \sigma a>0, \quad t \in \mathbb{R}
$$

Thus, the two almost periodic solutions $\mathfrak{u}_{1}, u_{2}$ are both positive solutions.

Interestingly, by applying Lemma 1.2, we can get a "better" result for equation (1.2).

Theorem 2.2. Assume that (H0)-(H4) hold. Then, there exists $\mathrm{L}^{*}>0$ such that equation (1.2) has at least three positive almost periodic solutions provided that $\mathrm{L}_{e}<\mathrm{L}^{*}$ and

$$
\lim _{r \rightarrow+\infty} \frac{\left\|\mu_{r}\right\|_{p}}{r}=0
$$


Proof. Since $\lim _{\mathrm{r} \rightarrow+\infty} \frac{\left\|\mu_{\mathrm{r}}\right\|_{\mathrm{p}}}{\mathrm{r}}=0$, one can choose $\sigma \in(0,1)$ such that $(2.1)$ holds and

$$
\sup _{t \in \mathbb{R}, x \in \mathbb{R}^{+}} e(t, x) \cdot\left[\|h\|+\xi\|m\|_{q}\left\|\mu_{\frac{c}{\sigma}}\right\|_{p}+M\|m\|_{q}\|g\|_{p}\right] \leqslant \frac{c}{\sigma} .
$$

Let $K$ and $\Phi$ be the same as in the proof of Theorem 2.1, i.e.,

$$
K=\left\{y \in A P(\mathbb{R}): \inf _{t \in \mathbb{R}} y(t) \geqslant \sigma\|y\|\right\},
$$

and

$$
(\Phi y)(t)=u_{y}(t)=e\left(t, u_{y}(t)\right)\left[h(t)+\int_{\mathbb{R}} k(t, s) f(s, y(s)) d s\right], \quad t \in \mathbb{R}, y \in \bar{K}_{c_{4}} .
$$

Then, it follows from the proof of Theorem 2.1 that $\Phi: \overline{\mathrm{K}}_{\mathrm{c}_{4}} \rightarrow \mathrm{K}$ is completely continuous.

Denote

$$
\mathrm{c}_{1}=\mathrm{b}, \quad \mathrm{c}_{2}=\mathrm{c}, \quad \mathrm{c}_{3}=\mathrm{c}_{4}=\frac{\mathrm{c}}{\sigma} .
$$

Then, $0<c_{1}<c_{2}<c_{3} \leqslant c_{4}$. Denote $\psi(u)=\inf _{t \in \mathbb{R}} u(t)$ for all $u \in k, t \in \mathbb{R}$. It is easy to see that $\psi$ is a concave nonnegative continuous functional on $\mathrm{K}$ and $\psi(\mathrm{u}) \leqslant\|u\|$.

For every $u \in \overline{\mathrm{K}}_{\mathrm{c}_{4}}$, we have $u \in \mathrm{K}$ and $\|\mathrm{u}\| \leqslant \mathrm{c}_{4}$. By (H1) and (2.2), we get

$$
\|\Phi(u)\| \leqslant \sup _{t \in \mathbb{R}, x \in \mathbb{R}^{+}} e(t, x) \cdot\left[\|h\|+\xi\|m\|_{q}\left\|\mu_{c_{4}}\right\|_{p}+M\|m\|_{q}\|g\|_{p}\right] \leqslant c_{4} .
$$

On the other hand, for every $u \in \overline{\mathrm{K}}_{\mathrm{c}_{4}}$, it follows from (2.1) that

$$
(\Phi u)(t) \geqslant \inf _{t \in \mathbb{R}, x \in \mathbb{R}^{+}} e(t, x) \cdot \inf _{t \in \mathbb{R}} h(t) \geqslant \sigma \sup _{t \in \mathbb{R}, x \in \mathbb{R}^{+}} e(t, x) \cdot\left[\|h\|+\|m\|_{q}\left(\xi\left\|\mu_{c_{4}}\right\|_{p}+M\|g\|_{p}\right)\right] \geqslant \sigma\|\Phi u\|
$$

for all $t \in \mathbb{R}$. Thus, we show that $\Phi$ maps $\overline{\mathrm{K}}_{\mathrm{c}_{4}}$ into $\overline{\mathrm{K}}_{\mathrm{c}_{4}}$. Similarly, for every $u \in \overline{\mathrm{K}}_{\mathrm{c}_{1}}$, since $\|\mathrm{u}\| \leqslant \mathrm{c}_{1}=\mathrm{b}$, we have $g(t, u(t)) \leqslant g(t)$ for all $t \in \mathbb{R}$, and

$$
\|\Phi(u)\| \leqslant \sup _{t \in \mathbb{R}, x \in \mathbb{R}^{+}} e(t, x) \cdot\left(\|h\|+\xi\|m\|_{q}\left\|\mu_{c_{1}}\right\|_{p}+\|m\|_{q}\|g\|_{p}\right)<b=c_{1},
$$

i.e., the condition (ii) of Lemma 1.2 holds. By the definition of $K$ and $c_{3}$, it is easy to see that the condition (iii) of Lemma 1.2 holds. In addition, for every $u \in K\left(\psi, c_{2}, c_{3}\right)$, we have $\inf _{t \in \mathbb{R}} u(t)=\psi(u) \geqslant c_{2}=c$, which means $g(t, u(t)) \geqslant \eta g(t)$ for all $t \in \mathbb{R}$, and thus by (H3)

$$
\begin{aligned}
\psi(\Phi u) & \geqslant \inf _{t \in \mathbb{R}, x \in \mathbb{R}^{+}} e(t, x) \cdot\left(\inf _{t \in \mathbb{R}} \int_{\mathbb{R}} k(t, s) f(s, u(s)) d s+\inf _{t \in \mathbb{R}} h(t)\right) \\
& \geqslant \inf _{t \in \mathbb{R}, x \in \mathbb{R}^{+}} e(t, x) \cdot\left(\eta \inf _{t \in \mathbb{R}} \int_{\mathbb{R}} k(t, s) g(s) d s+\inf _{t \in \mathbb{R}} h(t)\right)>c=c_{2} .
\end{aligned}
$$

This verifies (i) of Lemma 1.2. Then, it follows from Lemma 1.2 that $\Phi$ has at least three fixed points in $\overline{\mathrm{K}}_{\mathrm{c}_{4}}$, and thus equation (1.2) has at least three nonnegative almost periodic solutions $v_{1}, v_{2}, v_{3}$. Moreover, by Lemma 1.2, there holds

$$
\left\|v_{1}\right\| \leqslant \mathrm{b}<\left\|v_{2}\right\|, \quad \psi\left(v_{2}\right)<\mathrm{c}<\psi\left(v_{3}\right) .
$$

Noting that $\inf _{t \in \mathbb{R}} h(t)>0$ and $\inf _{t \in \mathbb{R}, x \in \mathbb{R}^{+}} e(t, x)>0$, we know that $v_{1}, v_{2}, v_{3}$ are not 0 . So

$$
v_{i}(t) \geqslant \sigma\left\|v_{i}\right\|>0, \quad i=1,2,3, t \in \mathbb{R},
$$

which means $v_{1}, v_{2}, v_{3}$ are three positive almost periodic solutions to equation (1.2). 
Remark 2.3. It is needed to note that the $\sigma$ in Theorem 2.2 is less than or equal to the $\sigma$ in Theorem 2.1. Thus, in most cases, the $L^{*}$ in Theorem 2.2 is less than or equal to the $L^{*}$ in Theorem 2.1. So we do not think that the assumptions of Theorem 2.2 are equal to the assumptions of Theorem 2.1.

Example 2.4. Let $e(t, x)=1+\lambda \sin ^{2}[x \cos t+x \cos \pi t], \lambda>0$ is a constant,

$$
\begin{aligned}
h(t) & =2+\cos ^{2} \pi t+\cos ^{2} t, & k(t, s) & =\frac{(2+|\cos \sqrt{2} t+\cos t|) e^{-s^{2}}}{4} \\
\tilde{f}(t, x) & =\frac{\sqrt{|x|} \sin ^{2}\left(x e^{t^{2}}\right)}{20\left(1+t^{2}\right)}, & g(t, x) & = \begin{cases}e^{-t^{2},} & x \leqslant 9, \\
(19 x-170) e^{-t^{2}}, & 9<x<10, \\
20 e^{-t^{2}}, & x \geqslant 10,\end{cases}
\end{aligned}
$$

and

$$
\mathrm{p}=1, \mathrm{q}=\infty, \xi=1, \eta=M=20, \mathrm{c}=10 .
$$

It is easy to see that $(\mathrm{H} 0)$ holds with $\mathrm{L}_{e} \leqslant 2 \lambda$. By some direct calculations, one can show that (H1) holds with

$$
\mu_{r}(t)=\frac{\sqrt{r}}{20\left(1+t^{2}\right)}, \quad g(t)=e^{-t^{2}}
$$

Moreover, we have

$$
\left\|\mu_{\mathrm{r}}\right\|_{1}=\frac{\pi}{20} \sqrt{r}, \quad \lim _{\mathrm{r} \rightarrow \infty} \frac{\left\|\mu_{\mathrm{r}}\right\|_{1}}{\mathrm{r}}=0 .
$$

By choosing $m(s)=e^{-s^{2}}$, (H2) holds. It follows from

$$
\inf _{t \in \mathbb{R}, x \in \mathbb{R}^{+}} e(t, x) \cdot\left[\eta \inf _{t \in \mathbb{R}} \int_{\mathbb{R}} k(t, s) g(s) d s+\inf _{t \in \mathbb{R}} h(t)\right] \geqslant 5 \sqrt{2 \pi}+2>10
$$

that (H3) holds. Let $b=9$. Then (H4) holds since

$$
\sup _{t \in \mathbb{R}, x \in \mathbb{R}^{+}} e(t, x) \cdot\left[\|h\|+\xi\|m\|_{q}\left\|\mu_{b}\right\|_{p}+\|m\|_{q}\|g\|_{p}\right] \leqslant(1+\lambda)\left(4+\frac{3 \pi}{20}+\sqrt{\pi}\right)<9=b,
$$

provided that $\lambda<\frac{9}{4+\frac{3 \pi}{20}+\sqrt{\pi}}-1$. By using Theorem 2.2, there exists $L^{*}>0$ such that equation (1.2) have three positive almost periodic solutions if $\lambda<\frac{\mathrm{L}^{*}}{2}$.

\section{Acknowledgment}

H.-S. Ding acknowledges support from the NSF of China, and the NSF of Jiangxi Province. The research of J. J. Nieto has been partially supported by the Ministerio de Economia y Competitividad of Spain under grant MTM2016-75140-P, and XUNTA de Galicia under grant GRC2015-004 and co-financed by the European Community fund FEDER.

\section{References}

[1] R. P. Agarwal, D. O'Regan, Existence theory for single and multiple periodic and almost periodic solutions of nonlinear integral equations, Nonlinear Oscil., 4 (2001), 2-15. 1, 1, 1

[2] E. Ait Dads, P. Cieutat, L. Lhachimi, Existence of positive almost periodic or ergodic solutions for some neutral nonlinear integral equations, Differential Integral Equations, 22 (2009), 1075-1096.

[3] E. Ait Dads, P. Cieutat, L. Lhachimi, Positive pseudo almost periodic solutions for some nonlinear infinite delay integral equations, Math. Comput. Modelling, 49 (2009), 721-739.

[4] E. Alvarez, C. Lizama, Weighted pseudo almost periodic solutions to a class of semilinear integro-differential equations in Banach spaces, Adv. Difference Equ., 2015 (2015), 18 pages. 
[5] E. Alvarez, C. Lizama, R. Ponce, Weighted pseudo antiperiodic solutions for fractional integro-differential equations in Banach spaces, Appl. Math. Comput., 259 (2015), 164-172.

[6] J. O. Alzabut, J. J. Nieto, G. T. Stamov, Existence and exponential stability of positive almost periodic solutions for a model of hematopoiesis, Bound. Value Probl., 2009 (2009), 10 pages. 1

[7] R. I. Avery, J. Henderson, Two positive fixed points of nonlinear operators on ordered Banach spaces, Comm. Appl. Nonlinear Anal., 8 (2001), 27-36. 1, 1.1

[8] A. Bellour, E. Ait Dads, Periodic solutions for nonlinear neutral delay integro-differential equations, Electron. J. Differential Equations, 2015 (2015), 9 pages. 1

[9] C. Corduneanu, Almost Periodic Functions, Second edition, AMS Chelsea Publishing, New York, (1989). 1, 1.8, 1.9

[10] H.-S. Ding, Y.-Y. Chen, G. M. N'Guérékata, $C^{n}$-almost periodic and almost periodic solutions for some nonlinear integral equation, Electron. J. Qual. Theory Differ. Equ., 2012 (2012), 13 pages. 1, 1

[11] H.-S. Ding, Q.-L. Liu, G. M. N'Guérékata, Equi-asymptotically almost periodic functions and applications to functional integral equations, Electron. J. Differential Equations, 2013 (2013), 9 pages. 1

[12] H.-S. Ding, Q.-L. Liu, J. J. Nieto, Existence of positive almost periodic solutions to a class of hematopoiesis model, Appl. Math. Model., 40 (2016), 3289-3297.

[13] H.-S. Ding, G. M. N'Guérékata, A note on the existence of positive bounded solutions for an epidemic model, Appl. Math. Lett., 26 (2013), 881-885. 1

[14] R. W. Leggett, L. R. Williams, Multiple positive fixed points of nonlinear operators on ordered Banach spaces, Indiana Univ. Math. J., 28 (1979), 673-688. 1, 1.2

[15] W. Long, W.-H. Pan, Asymptotically almost periodic solution to a class of Volterra difference equations, Adv. Difference Equ., 2012 (2012), 12 pages. 1, 1

[16] W. Long, X.-J. Zheng, L. Li, Existence of periodic solutions for a class of functional integral equations, Electron. J. Qual. Theory Differ. Equ., 2012 (2012), 11 pages. 1

[17] D. O'Regan, M. Meehan, Periodic and almost periodic solutions of integral equations, Appl. Math. Comput., 105 (1999), 121-136. 1, 1

[18] A. Sadrati, A. Zertiti, Existence and uniqueness of positive almost periodic solutions for systems of nonlinear delay integral equations, Electron. J. Differential Equations, 2015 (2015), 12 pages. 1 